\title{
Isolated Inferior Rectus Paresis in a Patient with a History of Lymphoma
}

\author{
Sung Hee Hwang, Seok-Beom Kwon, San Jung, Mi Jeong Lee, Ji Young Kim, \\ Suk Yun Kang, Ki Han Kwon
}

Can. J. Neurol. Sci. 2009; 36: 515-517

Isolated inferior rectus paresis due to a midbrain lesion is very uncommon. To the best of our knowledge, there are only three cases of infarction in the literature. ${ }^{1-3}$ It seems that selective oculomotor fascicular lesions may be more common than selective oculomotor nucleus lesions, possibly due to complicated perforating arteries ${ }^{4}$ or wider distribution in fascicular arrangement. ${ }^{5}$ The possible stroke etiology was small vessel disease..$^{1-3}$ We present a patient with a tiny infarction in the oculomotor nucleus. The lesion in this case is illustrative because it was very selective and only detected by brain diffusion-weighted magnetic resonance imaging (DWI). The patient had a history of previous transient ischemic attacks (TIAs) and Hodgkin's lymphoma with complete remission. In this case, the possible stroke mechanism may be different from that identified in previous reports.

\section{Case Report}

A 46-year-old woman with two previous TIAs was admitted for evaluation of painless diplopia and dizziness with acute onset. She did not have hypertension, diabetes mellitus, hyperlipidemia or a history of smoking, and had no family history of stroke. Fifteen years prior, she had been diagnosed with Hodgkin's lymphoma and treated with radiotherapy at a different hospital. She indicated that the radiation field included the neck and inguinal area, but not the head. She reported that there was no relapse with regular follow-ups. Her diplopia was more severe when she gazed to the right. The neurological examination was normal except for right isolated rectus paresis. The maximal limitation of right eye movement was associated with a right downward gaze (Figure A). Her right eye was not depressed on abduction and there was no ptosis. Pupils were symmetric and reactive to light stimulation. Nystagmus was not observed. She had no head tilt. A 1.5 Tesla MRI scan (Intera, Philips Medical Systems, Best, The Netherlands) was performed (5 mm slice thickness; $2.5 \mathrm{~mm}$ interslice gap; 23 axial slices; $230 \mathrm{~mm}$ field of view). Brain diffusion-weighted magnetic resonance imaging (DWI, TR 4,032 msec, TE $80 \mathrm{msec}$; matrix number of 192 x 192; two $\mathrm{b}$ values of 0 and $1,000 \mathrm{sec} / \mathrm{mm}^{2}$ ) and an apparent diffusion coefficient (ADC) map showed a focal infarct in the right median midbrain ventral to the aqueduct at the level of superior colliculus, presumably involving the right dorso-lateral subnucleus of the oculomotor nuclear complex (Figure B,C). Magnetic resonance angiography, routine laboratory tests, electrocardio-graphy, transthoracic (TTE) and transesophageal echocardiography (TEE) were performed with normal results. Additional laboratory tests were all normal and included analysis of protein $\mathrm{C}$, protein $\mathrm{S}$, antithrombin III, cryoglobulin, C3, C4, CH50, antinuclear antibody, lupus anticoagulant, anticardiolipin antibody ( $\operatorname{Ig~G}, \operatorname{Ig}$ M and Ig A), autoantibodies (anti-SM, anti-SSA, anti-ds-DNA, anti-RNP, anti-SS, anti-Scl-70 antibody, antinuclear cytoplasmic antibody), rheumatoid factor, protein electrophoresis and peripheral blood smear. Because of the frequent transient neurological deficits and the unusually small lesion, we evaluated the cerebrospinal fluid including the oligoclonal band, which was unremarkable. Visual evoked potential was normal. Her diplopia quickly improved to a nearly full recovery within nine days of admission, and was completely resolved twomonths after the onset of the diplopia with antiplatelet medication. During the seven-month follow-up period, she did not report any new events related to neurologic dysfunction.

\section{Discussion}

Isolated inferior rectus paresis due to a midbrain infarction is extremely rare. ${ }^{1}$ Our case is the first report of oculomotor nucleus infarction causing isolated inferior rectus paresis in the English literature..$^{1-3}$ The oculomotor nuclear complex is a collection of cell columns and discrete subnuclei that innervate the extraocular and levator palpebrae muscles to deliver preganglionic parasympathetic fibers to the ciliary ganglion. The dorsal cell column (dorso-lateral subnucleus) is situated in the most rostral portion, which supplies the inferior rectus. ${ }^{6}$ Considering the anatomical structure, selective involvement of this rostral area may cause isolated inferior rectus palsy without any other neurological deficit.

All previous cases showing inferior rectus paresis were found in patients with small vessel diseases. ${ }^{1-3}$ We failed to identify a possible etiology in spite of extensive investigation. It is known that radiation therapy accelerates atherosclerosis in the field of radiation ${ }^{7}$ and carotid disease frequently occurs after neck radiation for Hodgkin's lymphoma. ${ }^{8}$ A higher incidence of cardiac dysfunction following radiation therapy has been reported ${ }^{9}$. In our case, the result of MRA

From the Department of Neurology, Kang-Nam Sacred Heart Hospital, Hallym University College of Medicine (SHH, S-BK, SJ, MJL, JYK, SYK) and Department of Neurology, Hangang Sacred Heart Hospital, Hallym University College of Medicine (KHK), Seoul, Republic of Korea.

Received December 8, 2008. Final Revisions Submitted March 9, 2009. Correspondence to: Suk Yun Kang, Department of Neurology, Kang-Nam Sacred Heart Hospital, Hallym University College of Medicine, 948-1 Daerim-dong, Yeongdeungpo-gu, Seoul, 150-950, Republic of Korea. 

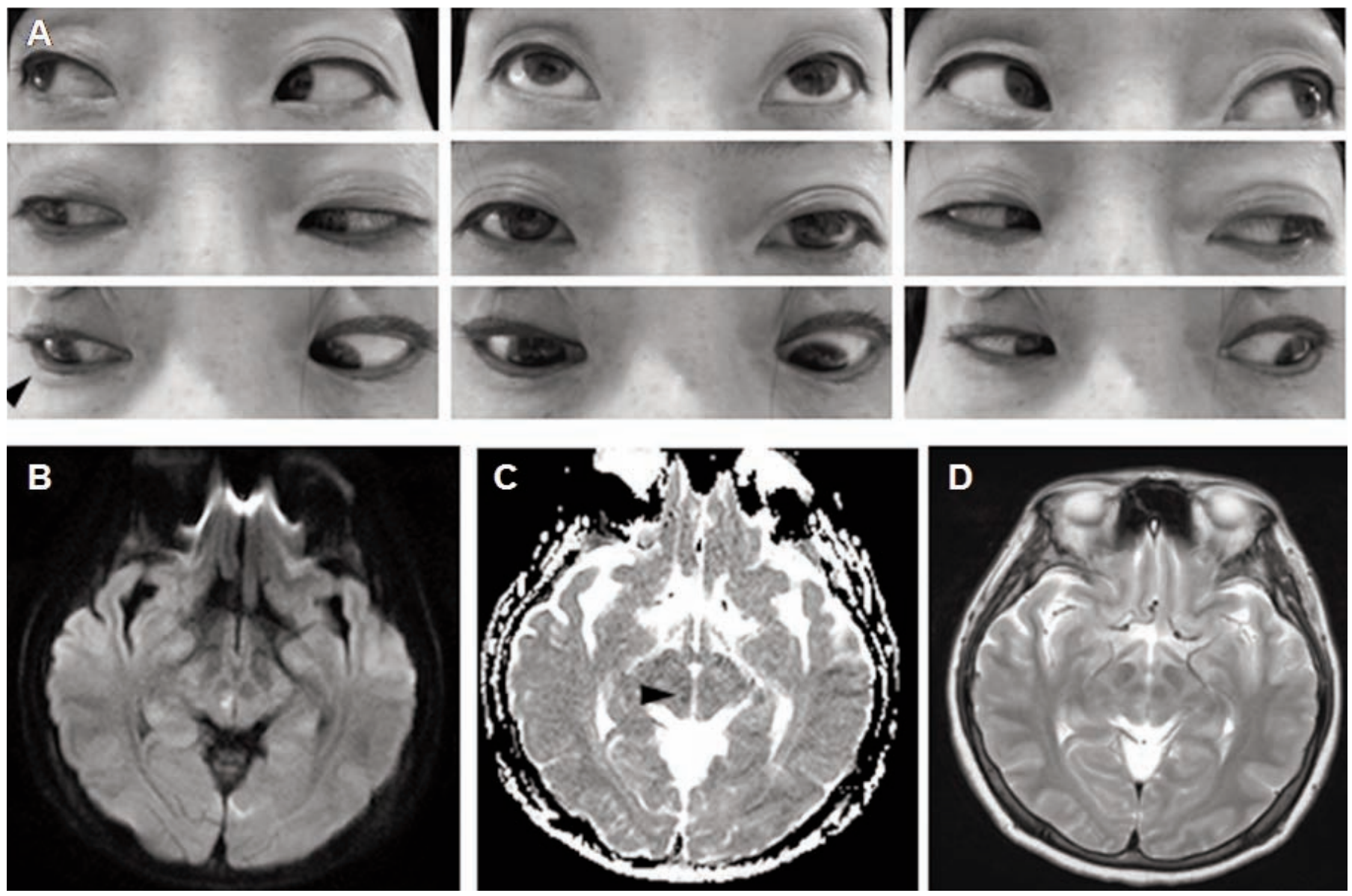

Figure: Extraocular movement and MRI findings. A. Photographs of extraocular movement show partial limitation of the right eye in the right downward direction (arrowhead). B. Diffusion-weighted MRI (DWI) reveals an acute tiny infarction in the right median midbrain ventral to the aqueduct at the level of superior colliculus. C. Apparent diffusion coefficient map shows hypointensity in the corresponding area of DWI (arrow). D. T2-weighted MRI (T2WI) does not show any definite abnormality.

and cardiac investigation including TEE were normal. We believe that an embolic mechanism of unknown origin could be the cause because of the frequent TIAs. Repeated ischemic attacks were reported in 14 young patients with remission of Hodgkin's lymphoma. ${ }^{10}$ Thirteen patients received radiotherapy and the other was treated with chemotherapy alone. Twelve of the patients had risk factors, but the remaining did not, and had normal angiographic findings and cardiac function in spite of frequent episodes like those in our case. The neurologic symptoms were visual symptoms, language disorders and sensory-motor deficits. It was postulated that the symptoms, possibly due to transient ischemia, might be a syndrome related to Hodgkin's lymphoma, but this hypothesis needs more investigation.

While the very small lesion was not easily seen on conventional MRI, it was clearly seen on both DWI and apparent diffusion coefficient map (Figure B,C). Sometimes ADC mapping is not helpful. ${ }^{1}$ The difference was only due to the time of MRI scanning after stroke, because the MRI parameters were similar in both studies. ${ }^{1}$ In our patient, the DWI was performed 24 hours after the onset of symptoms. In the previous case, the DWI was performed several days later. This observation suggests that the time-point for DWI/ADC map can affect the detection of cerebral infarction in cases of a very focal lesion.

In summary, isolated inferior rectus paresis may result from selective involvement of the most rostral area of the oculomotor nuclear complex. The time-point of brain DWI/ADC mapping could be important for determining the cause of acute isolated inferior rectus paresis. After a full investigation of our case, we were not able to determine a possible stroke mechanism, but we presume that the etiology is embolism of unknown origin. Additional work is needed to address the possible association between recurrent cerebrovascular attacks without large vessel diseases and Hodgkin's lymphoma treated with radiation therapy. 


\section{REFERENCES}

1. Lee DK, Kim JS. Isolated inferior rectus palsy due to midbrain infarction detected by diffusion-weighted MRI. Neurology. 2006;66(12):1956-7.

2. Negoro K, Sasabe F, Morimatsu M. [Isolated inferior rectus muscle paresis from midbrain infarction]. Rinsho Shinkeigaku. 1993;33 (4):434-6.

3. Takano M, Aoki K. [Midbrain infarction presenting isolated inferior rectus nuclear palsy]. Rinsho Shinkeigaku. 2000;40(8):832-5.

4. Thomke F, Gutmann L, Stoeter P, Hopf HC. Cerebrovascular brainstem diseases with isolated cranial nerve palsies. Cerebrovasc Dis. 2002;13(3):147-55.

5. Ksiazek SM, Slamovits TL, Rosen CE, Burde RM, Parisi F. Fascicular arrangement in partial oculomotor paresis. Am J Ophthalmol. 1994;118(1):97-103.
6. Parent A. Carpenter's human neuroanatomy. 9th ed. Philadelphia: Williams \& Wilkins; 1996.

7. Rogers LR. Cerebrovascular complications in cancer patients. Neurol Clin. 2003;21(1):167-92.

8. King LJ, Hasnain SN, Webb JA, Kingston JE, Shafford EA, Lister A, et al. Asymptomatic carotid arterial disease in young patients following neck radiation therapy for Hodgkin lymphoma. Radiology. 1999;213(1):167-72.

9. Hull MC, Morris CG, Pepine CJ, Mendenhall NP. Valvular dysfunction and carotid, subclavian, and coronary artery disease in survivors of Hodgkin lymphoma treated with radiation therapy. JAMA. 2003;290(21):2831-7.

10. Feldmann E, Posner JB. Episodic neurologic dysfunction in patients with Hodgkin's disease. Arch Neurol. 1986;43(12):1227-33. 